\title{
Profiles of disability among adults with bipolar spectrum disorders
}

\author{
Brian E. Perron • Amy S. B. Bohnert • \\ Michael G. Vaughn • Mark S. Bauer • \\ Amy M. Kilbourne
}

Received: 29 September 2008/ Accepted: 1 October 2009

(C) Springer-Verlag 2009

\begin{abstract}
Background Heterogeneous groups of patients with a spectrum of service needs are commonplace in mental health settings. Although comprehensive assessments are available to measure variations in service needs, numerous challenges still exist when confronting this heterogeneity and many assessments used in clinic settings are lengthy and have not been demonstrated to be consistent over time. Objective The purpose of this study was to identify subgroups of persons with bipolar spectrum disorders, who have similar disability profiles, and to the extent to which the subgrouping is stable over time.

Methods Participants were recruited from the Continuous Improvement for Veterans in Care-Mood Disorders. Eligible patients $(N=435)$ were those who received inpatient or outpatient treatment for bipolar disorder at a large urban VA mental health facility in Western Pennsylvania from July 2004 through July 2006. This was a naturalistic cohort study of patients with bipolar spectrum disorders. Baseline and 1-year follow-up data were collected using face-to-face interviews and recorded abstraction. The World Health Organization Disability Assessment Scale was the primary measure used to identify subgroups within this sample.

Results Using a classification strategy called latent profile analysis, this study identified three unique subgroups that
\end{abstract}

B. E. Perron $(\square)$ · A. S. B. Bohnert · A. M. Kilbourne

University of Michigan, Ann Arbor, USA

e-mail: beperron@umich.edu

M. G. Vaughn

Saint Louis University, St. Louis, MO, USA

M. S. Bauer

VA Boston Healthcare System \& Harvard Medical School,

Boston, MA, USA showed significant differences in various clinical measures at baseline and follow-up. The largest and most consistent subgroup differences were observed in the current bipolar symptomatology.

Conclusion The classification of functional status in the present study can aid clinicians in the identification of bipolar patients, with specific impairment profiles, who may need additional intervention. Future research is needed to understand whether specific interventions targeted at these subgroups can improve the quality of care for this high-need and at-risk population.

Keywords Bipolar disorders · Disability ·

Classification strategies $\cdot$ Latent profile analysis

\section{Introduction}

Serious mental illnesses (SMI), including schizophrenia, bipolar disorder and major depression, are the leading causes of functional decline and quality-adjusted life years that are lost (1998). Persons with SMIs are more likely to die younger and have poor social, functional and economic outcomes [6]. To address the risk of adverse outcomes, persons with SMI often need support for multiple needs beyond their psychiatric care (medical, housing, social, functional). Identifying specific subtypes of impairment and functioning can aid in the provision of appropriate services.

Heterogeneous groups of patients with a spectrum of service needs are commonplace in mental health settings [31]. Although comprehensive assessments are available to measure variations in service needs, numerous challenges still exist when confronting this heterogeneity and many assessments used in clinic settings are lengthy and have not been demonstrated to be consistent over time. When 
providers are confronted with multiple service needs, they must effectively prioritize a range of treatment and interventions that target service needs, which place competing demands on the individual [25]. Ultimately, accurate, yet practical, assessments of service needs are needed to effectively characterize the service needs of this population and develop programs that can address their range of needs. For persons with SMIs, tools to identify groups based on their functioning can aid in this process to reduce the burden of disease associated with these illnesses.

To date, research on service needs has been limited to descriptive assessments that may not capture the heterogeneity of vulnerable patient populations. There has been a growing interest in applying classification-based strategies, including cluster analysis, latent class analysis and latent profile analysis to identify discrete and fairly homogeneous subgroups within larger heterogeneous populations. Marias et al. (2008) argues that such type of analyses are especially useful for programs that are intended to service individuals with multiple co-occurring disorders or dual diagnoses. A notable example of this application is a cluster analysis of runaway homeless youth based on various high-risk behaviors [12]. These clusters were used to inform the matching needs to services, while reducing service redundancies. Other recent work involving classification-based analyses have examined service needs among persons in outpatient substance abuse treatment [33], behavioral problems in children who witness domestic violence [37], medical utilization patterns [18], substance involvement and criminal behavior [34], self-injurious behaviors [26] and psychosislike symptoms [35]. While these studies represent different problem areas and systems of care, they share a common objective of characterizing heterogeneous clinical populations to tailor intervention strategies by subgroups [1].

In following this line of research, the purpose of this study was to identify subgroups of persons with bipolar disorder, who have similar disability profiles, and to the extent to which the subgrouping is stable over time. This study was intended to characterize the service needs of a heterogeneous population and guide treatment planning, and to identify individuals who might be at risk for psychiatric relapse or other adverse outcomes. This study builds on the broader literature on the consequences of bipolar disorders. In addition to being the first study to identify subgroups of bipolar patients based on disability profiles, this is the first study, to our knowledge, that uses prospectively collected, longitudinal data to look at subgroups over time.

Bipolar spectrum disorders represent an ideal target population for the study of disability profiles. This disorder affects up to $5.5 \%$ of the US population [19] and is associated with a disproportionate burden of medical and substance use comorbidity [23, 30], health-care costs [36], complex patterns of service utilization [22] and social outcomes including homelessness and incarceration [6]. Few effective interventions have been developed to manage the complexity of this condition [6]. Prior research suggests that patients with bipolar disorder have more limited functioning compared to individuals with other mental illnesses $[11,21]$. For patients hospitalized with a manic episode, functioning shows less improvement than symptoms [14]. Among bipolar spectrum patients, minority status, cognitive abilities, substance abuse, psychosis and anxiety [13, 20], among other factors, are associated with poorer functioning. Additionally, evidence suggests that disability and functioning impact the course of illness of bipolar patients $[2,5,16,39]$, as disability predicts later episodes and symptoms [7]. While the study was exploratory in nature and consequently there were no formal hypotheses, subgroups of patients with common social, functional and health disabilities, identified by the analysis, will allow outpatient treatment programs to more efficiently structure services to help address multiple service needs.

\section{Methods}

\section{Data source}

Participants were recruited from the Continuous Improvement for Veterans in Care-Mood Disorders (CIVIC-MD) naturalistic cohort study of patients with bipolar disorder; additional details of this project, the study population and patient characteristics are provided elsewhere [24]. The aim of CIVIC-MD was to examine patient and provider factors associated with treatment quality and outcomes, along with important mediators of these outcomes within a routine care setting. Eligible patients were those who received inpatient or outpatient treatment for bipolar disorder at a large urban VA mental health facility in Western Pennsylvania from July 2004 through July 2006. Inclusion criteria included a current diagnosis of bipolar disorder (I, II, NOS), cyclothymia or schizoaffective disorder, bipolar subtype, based on chart review. For those patients who had a current diagnosis of one of these disorders, the patient's provider was approached for confirmation of the diagnosis. The primary exclusion criterion was having an unstable medical condition or significant cognitive impairment precluding patients from completing the surveys or providing informed consent. In total, the study staff approached 720 potential participants whose diagnosis was confirmed by their provider. Of these, 104 were excluded due to acute symptomatology, 148 refused to participate and 33 did not complete the baseline survey. The resulting sample included 435 individuals, 334 of whom were reassessed 1 year later. The majority (74\%) were diagnosed with bipolar I disorder, $2 \%$ with bipolar II, $7 \%$ with bipolar 
not otherwise specified, and $17 \%$ with schizoaffective disorder, bipolar subtype. Patients self-completed a survey that included questions regarding demographics and other patient characteristics, symptomatology, substance use, behavioral factors, functioning and treatment adherence. The study was reviewed and approved by the institutional review board of the medical center. Participants then selfcompleted a follow-up survey approximately 1 year later that comprised similar questions as the baseline survey (symptomatology, substance use, behavioral factors, functioning, and treatment adherence).

\section{Classification analysis}

\section{Disability indicators}

This study identified subgroups of persons using measures from the WHO Psychiatric Disability Schedule (WHODAS). This is a 12-item screening measure that assesses six domains of functioning: understanding and communicating, getting around, self-care, getting along with others, household and work activities, and participation in society (see Table 2 for a complete summary of items). This brief measure was chosen as the WHODAS is already commonly used to identify patient needs, problem-service matching and monitoring outcomes. Prior research has found the WHODAS to have high internal consistency, good test-retest reliability and evidence for validity [32].

\section{Analytic strategy}

Latent profile analysis (LPA) was used to explore different subgroups. Latent profile analysis is a statistical procedure for findings subgroups measured on continuous data. The results can be used to assign subjects to their most likely subgroup based on the observed data, similar to latent class analysis.

The analyses were carried out in an exploratory fashion using Mplus (version 4.2). We examined the fit of a series of different LPA models, starting with a model specifying a single group. Additional groups were added to the model until no significant empirical and conceptual improvements were observed. The empirical fit of the model was based on the Bayesian Information Criterion (BIC), with lower values reflecting an improved fit. Entropy was also examined, which shows how well the indicators predict subgroup membership, with values closer to 1.0 indicating better prediction. Finally, the conceptual fit of each model was examined using the model results, model diagnostics and a visual representation of subgroups. After finding a set of subgroups that exhibited a good empirical and conceptual fit with the data, the stability of the subgroup was tested using 1-year follow-up data. The same criteria for examining the fit of the baseline model were also used for examining the fit of the follow-up model. The $P$ value was set at 0.05 for all significance testing.

\section{Model validation}

After baseline and follow-up models were developed, subjects were assigned to a group based on their highest probability of membership as indicated by the model. Bivariate and multivariate analyses were conducted to examine the associations between clinical and psychosocial measures and functioning. Key variables hypothesized to be most influential included sociodemographics, bipolar disorder symptoms, substance use, suicidality, comorbidities and patient sociodemographics.

\section{Sociodemographics}

A set of demographic factors was also included: age (in years), ethnicity (non-Hispanic black, Hispanic, and nonHispanic non-black), education (in years) and gender (male/female).

\section{Bipolar disorder symptoms}

The Internal States Scale (ISS) is a 15-item measure designed to elicit self-report of current mood symptoms, which are used to assign current mood episode status (hypo)mania, depression, mixed state or euthymia (normal mood). For each item, the participants rate how strongly they agree with such statements as "today I feel depressed" and "today I feel like a capable person" on a scale of 0 (not at all) to 100 (completely). The ISS consists of four empirically defined and cross-validated subscales $[3,4,10]$ : activation (5 items, range $0-500$ ), correlated with clinician ratings of current manic symptoms and used in the algorithm to assign current mood episode status; the Depression Index ( 2 items, range 0-200), correlated with clinician ratings of depressive symptoms; well-being ( 3 items, range $0-300$ ), used in the algorithm to assign current mood episode status; and perceived conflict (5 items, range 0-500), correlated with clinician ratings of global psychopathology. The total score of each subscale is the sum of its items, so that a higher score represents a greater sense of well-being, depression, personal conflict or activation/agitation.

\section{Substance use}

Participants were asked to report the use of substances in the previous year. Alcohol use was measured on a five-point ordinal scale, ranging from never used to more than four times a week, which is indicative of hazardous drinking [17]. Other illicit drug use, including marijuana, cocaine, 
hallucinogens, opiates or stimulants, was measured on a four-point scale (have never tried to every day). Participants were also asked the extent to which drugs interfered with work at school, job or at home. Responses were recorded on a five-point scale (once or twice to more than 20 times).

\section{Suicidality}

Suicidal ideation was measured using a single item from the Patient Health Questionnaire (PHQ-9) [27]. Patients were asked how often in the previous 2 weeks they had "thoughts that you would be better off dead or of hurting yourself in some way", scored from " 0 " (not at all) to " 3 " (nearly every day). The PHQ-9 exhibits good reliability, convergent/discriminant validity and responsiveness to change in recent research [9].

\section{Comorbidities}

The number of medical conditions based on medical record review was also included using a standardized form [15]. In addition, patients' self-reported anxiety symptoms were included, using the anxiety measurement of the PRIMEMD [38].

\section{Results}

Summary of sample characteristics

The mean age of the study population was 49.4 years $(\mathrm{SD}=10.6)$, with $14 \%$ women and $23 \%$ ethnic minorities (including 13\% African-Americans). This sample was representative of veterans diagnosed with bipolar disorder [8]. Approximately, $66 \%$ had some college education; $28 \%$ of the sample reported some drug use in the previous year and $21 \%$ reported hazardous drinking in the previous year. As much as $30 \%$ had had a recent manic episode and $12 \%$ reported being homeless.

\section{Summary of disability}

Table 1 provides descriptive and bivariate summary of impairments across all 12 items measured at baseline. Follow-up data were nearly identical and excluded for purposes of brevity, but available on request from the first author. Two items reflecting self-care, i.e., washing your whole body and getting dressed, had the lowest mean group scores (low disability). Disabilities that reflected psychosocial functioning had the highest scores (high disability), especially being emotionally affected by health problems and hesitancy joining in community activities. All disability indicators exhibited small to medium inter-correlations, ranging from 0.20 to 0.66 , suggesting that indicators reflect different aspects of disability with some shared variance, but no inter-correlations were so high as to indicate redundancy in measurement.

Latent profile analysis

An exploratory search for subgroups of patients with similar disability profiles was performed using latent profile analysis (LPA). A total of four models were examined using the baseline data, with one to four classes. The

Table 1 Descriptive summary and bivariate associations among WHODAS items at baseline

\begin{tabular}{|c|c|c|c|c|c|c|c|c|c|c|c|c|}
\hline Impairment indicator $^{\mathrm{a}}$ & $\begin{array}{l}\text { Baseline mean } \\
\text { (SD) }\end{array}$ & 1. & 2. & 3. & 4. & 5. & 6. & 7. & 8. & 9. & 10. & \\
\hline 1. Standing for long periods of time, such as $30 \mathrm{~min}$ & $1.4(1.3)$ & - & & & & & & & & & & \\
\hline 2. Taking care of your household responsibilities & $1.4(1.2)$ & 0.39 & - & & & & & & & & & \\
\hline 3. Learning a new task & $1.1(1.2)$ & 0.23 & 0.47 & - & & & & & & & & \\
\hline 4. Joining in community activities & $1.7(1.4)$ & 0.26 & 0.49 & 0.42 & - & & & & & & & \\
\hline 5. Being emotionally affected by your health problems & $1.8(1.2)$ & 0.44 & 0.46 & 0.39 & 0.48 & - & & & & & & \\
\hline 6. Concentrating on doing something for $10 \mathrm{~min}$ & $1.4(1.2)$ & 0.29 & 0.43 & 0.50 & 0.45 & 0.49 & - & & & & & \\
\hline 7. Walking a long distance, such as a mile & $1.5(1.5)$ & 0.62 & 0.33 & 0.20 & 0.27 & 0.33 & 0.25 & - & & & & \\
\hline 8. Washing your whole body & $0.54(0.95)$ & 0.35 & 0.40 & 0.29 & 0.26 & 0.28 & 0.37 & 0.40 & - & & & \\
\hline 9. Getting dressed & $0.48(0.83)$ & 0.37 & 0.42 & 0.38 & 0.24 & 0.36 & 0.38 & 0.41 & 0.66 & - & & \\
\hline 10. Dealing with people you do not know & $1.4(1.3)$ & 0.30 & 0.38 & 0.44 & 0.56 & 0.45 & 0.54 & 0.23 & 0.30 & 0.34 & - & \\
\hline 11. Maintaining a friendship & $1.4(1.3)$ & 0.20 & 0.39 & 0.42 & 0.60 & 0.45 & 0.48 & 0.13 & 0.24 & 0.29 & 0.62 & - \\
\hline 12. Completing your day-to-day work & $1.5(1.3)$ & 0.34 & 0.61 & 0.49 & 0.49 & 0.52 & 0.57 & 0.28 & 0.40 & 0.48 & 0.53 & 0.55 \\
\hline
\end{tabular}

a All items were preceded by the question, "In the last 30 days, how much difficulty did you have in ..." and rated on a five-point Likert-type scale $(0=$ not at all, $4=$ very much). Bivariate associations are presented as Pearson product moment correlations. Baseline data are presented below the main diagonal, and follow-up data above the main diagonal. All associations are statistically significant $(P<0.001)$ 
Table 2 Empirical fit and class sizes of baseline and follow-up models using latent profile analysis

\begin{tabular}{|c|c|c|c|c|c|c|}
\hline \multirow{2}{*}{$\begin{array}{l}\text { Number of } \\
\text { classes }\end{array}$} & \multicolumn{2}{|l|}{$\mathrm{BIC}$} & \multicolumn{2}{|l|}{ Entropy } & \multicolumn{2}{|c|}{ Class size $N(\%)$} \\
\hline & Baseline & Follow-up & Baseline & Follow-up & Baseline & Follow-up \\
\hline 1 & - & - & - & - & $418(100)$ & 315 (100) \\
\hline \multirow[t]{2}{*}{2} & 20,897 & 15,786 & 0.91 & 0.91 & $201(48.0)$ & $154(49.0)$ \\
\hline & & & & & $217(52.0)$ & $161(51.0)$ \\
\hline \multirow[t]{3}{*}{3} & 20,439 & 15,369 & 0.92 & 0.93 & $177(42.2)$ & $123.6(39.2)$ \\
\hline & & & & & $167(39.9)$ & $108.8(34.5)$ \\
\hline & & & & & 75 (17.9) & $82.6(26.2)$ \\
\hline \multirow[t]{4}{*}{4} & 20,368 & 15,321 & 0.93 & 0.93 & $176(42.1)$ & $124(39.4)$ \\
\hline & & & & & $165(39.5)$ & $102(32.4)$ \\
\hline & & & & & $20(4.8)$ & $19(6.0)$ \\
\hline & & & & & 57 (13.6) & $70(22.2)$ \\
\hline
\end{tabular}

empirical fit of these models and the estimated sizes of their subgroups are summarized in Table 2. A decrease in the BIC and increase in entropy were observed across all models when additional subgroups were added to the model, although the magnitude of differences was comparatively small. The conceptual fit of the models was examined by plotting the estimated mean values for each disability indicator for each subgroup. The most complex model (that is, the model with the four-group solution) appeared to have a good conceptual fit. However, the fourgroup solution included a subgroup with only $4.8 \%$ of subjects. This subgroup was small and exhibited a similar disability profile in comparison to another subgroup. The model with three subgroups showed clear differences across subgroups and had a more even distribution in the sizes of subgroups. Thus, the three-group solution was selected based on good empirical and conceptual fit. This entire process was repeated with the data at the 1-year follow-up, which also resulted in a model with three subgroups, with nearly identical disability profiles compared to the baseline data.

The estimated mean values for each impairment indicator across the three subgroups based on baseline data is depicted graphically in Fig. 1, although it is also representative of the follow-up data. The distinguishing features of each subgroup were used for selecting a suitable subgroup name. Group 1 represented the largest subgroup, exhibiting the lowest scores on all indicators. Thus, it is called the "low global disability" group. The second group had higher scores than the low disability group on physical and cognitive functioning, but the most pronounced differences were on the measures of psychosocial functioning. This group is referred to as the "high psychosocial disability" subgroup. The third group, which was the smallest in both the baseline and follow-up data, exhibited comparatively high scores on all indicators. Therefore, this group is referred to as the "high global disability" group. The "high psychosocial disability" group may also describe those

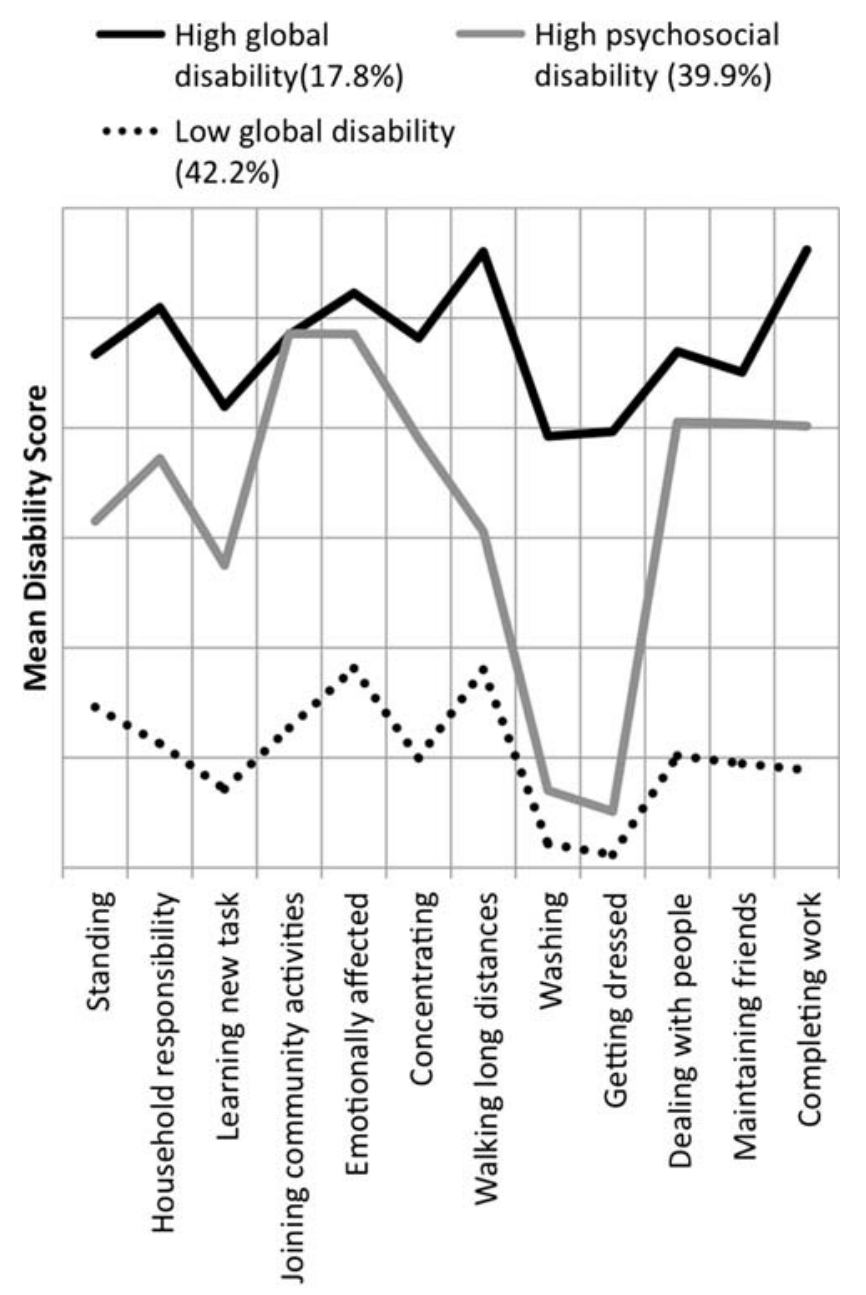

Disability Indicators

Fig. 1 Disability subgroups and mean scores on disability indicators among persons with bipolar disorder

individuals whose overall disability is moderate, as the group had scores that were substantially above the "low global disability" group, but below the "high global disability" group on some, but not all, measures. 
Class changes

A three-class solution was stable from baseline to followup in terms of disability profiles, including the mean values on the disability indicators. Further analyses were conducted to determine how many people changed from one subgroup to another over the following year. From baseline to follow-up, $66 \%$ of the subjects remained in the same disability class. Only $4 \%$ of the subjects changed from the low global disability subgroup to the high psychosocial disability class, or vice versa. The majority of the changes involved movement between the high psychosocial and high global disability class, and between the low disability and high psychosocial disability class. When the disability classes are viewed as ordered categorical variables, $11 \%$ of subjects moved from a lower to a higher disability subgroup, and $20 \%$ of subjects moved from a higher to a lower disability subgroup (Table 3).

Cross-sectional and longitudinal analysis

\section{Bivariate associations}

A series of correlations between baseline measures and disability subgroups was computed and is summarized in Table 4. Correlations with follow-up measures are similar and are therefore excluded for purposes of brevity. Age was the only demographic variable significantly associated with subgroup assignment, with post hoc contrasts indicating that persons in the low global disability subgroup

Table 3 Bivariate associations among disability classes and psychosocial and clinical variables at baseline

\begin{tabular}{|c|c|c|c|c|}
\hline Variable & $\begin{array}{l}\text { Low global } \\
\text { disability } \\
M(\mathrm{SD})\end{array}$ & $\begin{array}{l}\text { High psychosocial } \\
\text { disability } \\
M(\mathrm{SD})\end{array}$ & $\begin{array}{l}\text { High global } \\
\text { disability } \\
M(\mathrm{SD})\end{array}$ & $\begin{array}{l}\text { Test statistic } \\
F(P \text { value })\end{array}$ \\
\hline ISS well-being & $185.2(70.3)^{\mathrm{a}, \mathrm{b}, \mathrm{c}}$ & $135.4(76.9)^{\mathrm{a}, \mathrm{b}, \mathrm{c}}$ & $109.5(63.8)^{\mathrm{a}, \mathrm{b}, \mathrm{c}}$ & $33.0(<\mathbf{0 . 0 0 1})$ \\
\hline ISS, activation & $132.5(116.6)^{\mathrm{a}, \mathrm{b}}$ & $217.4(124.9)^{\mathrm{a}}$ & $232.2(131.5)^{\mathrm{b}}$ & $25.3(<\mathbf{0 . 0 0 1})$ \\
\hline ISS, depression & $45.0(50.8)^{\mathrm{a}, \mathrm{b}}$ & $95.3(57.7)^{\mathrm{a}}$ & $109.7(57.3)^{\mathrm{b}}$ & $48.3(<\mathbf{0 . 0 0 1})$ \\
\hline ISS, personal conflict & $71.9(82.9)^{\mathrm{a}, \mathrm{b}}$ & $167.5(120.5)^{\mathrm{a}}$ & $178.8(121.8)^{\mathrm{b}}$ & $41.1(<\mathbf{0 . 0 0 1})$ \\
\hline Age & $48.0^{\mathrm{a}}(11.1)$ & $53.0^{\mathrm{a}}(9.9)$ & $49.0(10.2)$ & $6.81(0.001)$ \\
\hline \multirow[t]{2}{*}{ Medical comorbidities } & $2.6(2.1)$ & $2.8(1.9)$ & $3.1(2.3)$ & $1.23(0.29)$ \\
\hline & $N(\%)$ & $N(\%)$ & $N(\%)$ & $\chi^{2}(P$-value $)$ \\
\hline \multicolumn{5}{|l|}{ Education } \\
\hline$<$ College & $58(32.6)$ & $50(30.1)$ & $30(40.5)$ & $2.54(0.281)$ \\
\hline$\geq$ College & $120(67.4)$ & $116(69.9$ & $44(59.5)$ & \\
\hline \multicolumn{5}{|l|}{ Race } \\
\hline White & $141(79.2)$ & $123(74.1)$ & $60(81.1)$ & $2.23(0.693)$ \\
\hline Black & $21(11.8)$ & $24(14.5)$ & $9(12.2)$ & \\
\hline Other & $16(9.0)$ & 19 (11.4) & $5(6.8)$ & \\
\hline \multicolumn{5}{|l|}{ Gender } \\
\hline Male & $157(88.2)$ & $138(83.1)$ & $62(83.8)$ & $1.96(0.375)$ \\
\hline Female & $21(11.8)$ & $28(16.9)$ & $12(16.2)$ & \\
\hline \multicolumn{5}{|c|}{ Drug use in the previous year } \\
\hline No & $136(76.4)$ & $110(66.3)$ & $54(73.0)$ & $4.42(0.110)$ \\
\hline Yes & $42(23.6)$ & $56(33.7)$ & $20(27.0)$ & \\
\hline \multicolumn{5}{|c|}{ Binge drinking in the previous year } \\
\hline No & $153(86.0)^{\mathrm{a}, \mathrm{b}}$ & $118(71.5)^{\mathrm{a}}$ & $55(74.3)^{\mathrm{b}}$ & $11.25(\mathbf{0 . 0 0 4})$ \\
\hline Yes & $25(14.0)$ & $47(28.5)$ & $19(25.7)$ & \\
\hline \multicolumn{5}{|l|}{ Suicidal ideation } \\
\hline No & $130(73.0)^{\mathrm{a}, \mathrm{b}}$ & $60(36.6)^{\mathrm{a}}$ & $20(27.4)^{b}$ & $64.45(<\mathbf{0 . 0 0 1})$ \\
\hline Yes & $48(27.0)$ & $104(63.4)$ & $53(72.6)$ & \\
\hline \multicolumn{5}{|l|}{ Anxiety } \\
\hline No & $86(48.3)^{\mathrm{a}, \mathrm{b}}$ & $24(14.5)^{\mathrm{a}}$ & $17(23.0)^{\mathrm{b}}$ & $48.88(<\mathbf{0 . 0 0 1})$ \\
\hline Yes & $92(51.7)$ & $142(85.5)$ & $57(77.0)$ & \\
\hline
\end{tabular}

All percentages are reported as column percentages unless noted otherwise. Superscripts identify classes that are statistically significant $(P<0.05)$ based on post hoc comparisons 


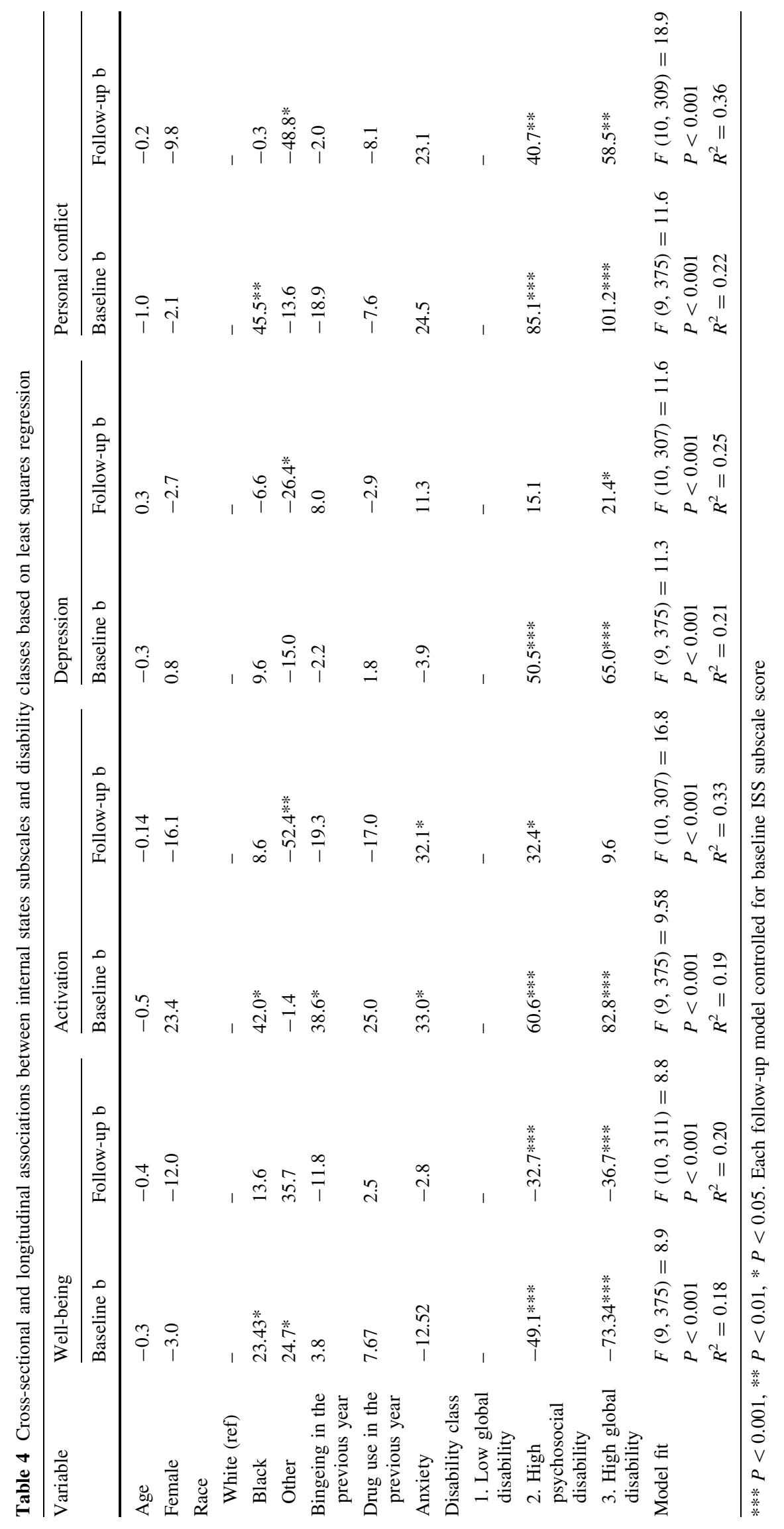


were significantly younger than subjects in the high psychosocial disability subgroup. Several clinical variables were significantly associated with subgroup assignment, all indicating that more severe or complex clinical profiles were associated with higher levels of symptomatology. These include anxiety, suicidal ideation, binge drinking in the previous year and all four ISS subscales. Post hoc contrasts revealed that high psychosocial and high global disability subgroups were not significantly different on these measures, with the exception of ISS well-being.

\section{Multivariate associations}

A series of ordinary least squares regression models were conducted to examine the associations between disability subgroups' baseline and follow-up ISS scores, while controlling for other potentially confounding variables (measured at baseline). It should be noted that each regression model included the respective ISS subscale baseline values as a control. Including all ISS subscales in each model was not possible due to their high inter-correlations (range of Pearson $r=|0.12|$ to $|0.72|$ ). Regarding the baseline models, each model was statistically significant. Of all the variables included in the model, disability class exhibited the strongest and most consistent association with each ISS outcome measure. A graded relationship was observed across the classes, with the high global disability exhibiting the strongest associations. Disability class was significantly associated with ISS subscale scores at the 1-year follow-up. The strongest and most consistent associations were observed on ISS measures, with the high global disability having the highest perceived conflict scores and lowest well-being scores. High psychosocial disability was associated with higher activation scores in comparison to low global disability, but high global disability was not. Conversely, high global disability was associated with higher depression scores in comparison to low global disability, but high psychosocial disability was not.

\section{Discussion}

Using the classification strategy, this study identified three unique subgroups of patients with bipolar disorder who have similar profiles of disability. These subgroups included low disability, high psychosocial disability and high global disability. Significant age differences were observed, with younger persons having the greatest likelihood of being in the low disability subgroup. No other significant differences were observed on sociodemographic measures, but subgroups differed on multiple clinical measures, especially bipolar symptomatology. Persons in both the moderate to high disability subgroups (i.e., psychosocial and global) exhibited significant associations with all bipolar symptom measures at baseline, but weaker associations with manic and depressive symptoms at the 1-year follow-up. These findings suggest that traditional bipolar symptom scales may not accurately reflect global disability, especially as they relate to global functioning over time.

Although almost $40 \%$ of the subjects changed subgroups from baseline to follow-up, only a small number of subjects moved to the low global disability group. Thus, this shows the durability of problems associated with psychosocial functioning and some changes in other areas of independent living skills and activities of daily living. This also provides further evidence that current treatment strategies, when focused primarily on reduction of manic and depressive symptoms, may not be sufficient to improve functioning.

From a treatment perspective, using these disability profiles in interpreting the WHODAS can aid in the assessment of domains of functioning that should be actively addressed in treatment planning for persons with bipolar disorders. The findings suggest that two types of specialized service options should be offered: (1) services that focus on improving psychological and social functioning, and (2) services that focus on improving physical functioning. Individuals who appear to fit the "high psychosocial disability" subgroup profile based on their WHODAS responses could be referred for the first type of services, while individuals who appear to fit the "high global disability" subgroup could be recommended for both types of services. The degree of instability of the disability group classifications over time also suggests that frequent re-assessment of disability is necessary.

This study also suggests a possible limitation of using the global disability rating of the WHODAS, computed as a summary score across individual items. Prior research shows internal consistency among the estimates, and the subgroups defined by the LPA largely represented levels of severity of disability. However, we also found that some subgroups of persons can exhibit high levels of disability on certain items and low levels on others. Summary of WHODAS scores may particularly misrepresent the treatment needs of individuals in the "high psychosocial disability" group, who had moderately high ratings of impairment when averaged across domains of functioning, but had low ratings of physical impairment with high ratings of psychological and social impairment.

There are limitations to this study that warrant consideration. First, CIVIC-MD was limited to a single site, and we were unable to enroll veterans who did not use any VA mental health care between July 2004 and July 2006. Second, to reduce respondent and provider burden and reduce barriers to study entry, we relied on the clinician diagnosis on record rather than on the formal structured 
psychiatric interview. While structured psychiatric interviews (e.g., structured clinical interview for DSM-IV) can be lengthy and potentially burdensome to patients, they are considered the gold standard and may preclude our ability to fully compare the results from CIVIC-MD with results from other clinical cohorts that implemented formal diagnostic assessment. Third, while we enrolled the majority of eligible patients, we were unable to enroll some who were too acutely ill or unable to participate because of lack of time. Yet, while enrolling patients with acute psychiatric symptoms may have led to an exclusion of sicker individuals, enrolling these patients may have been infeasible due to concerns regarding the ability of these patients to provide informed consent. Finally, it is important to recognize that the disability profiles were based only on the WHODAS. Other disability or impairment measures, such as the RIFT (Range of Impaired Functioning Tool; [29] and the Sheehan Disability Scale [28], may produce different profiles based on the specific areas that are assessed. Comparative studies using this classification methodology with different disability measures can be useful in determining the validity of the disability profiles. Classification using measures designed specifically for individuals with psychopathology (e.g., RIFT) could serve several purposes. These include indicating if specialized scales are more sensitive to patterns of impairment for individuals with bipolar disorder and determining the necessity of distinguishing impairments that are a direct result of symptomatology from those that are not, to understand disability in this population.

Despite these limitations, this study contributes to both the broader literature on the classification of risk factors and service needs among a vulnerable population. The results are similar to other studies that have found a small number of subgroups within a heterogeneous population $[12,33]$. These results warrant further investigation of this approach to confirm: the validity of the subgroups identified in this study; how subgroups groups may change when different disability measures or concepts are used as the basis of classification; and how specific types of treatment may shift patients from the higher to the lower disability groups. The results of classification-based strategies can also be integrated into research on service planning, to determine the potential costs and benefits of planning and delivering services to different subgroups. This can be done by addressing similar disabilities within each subgroup and exploring ways of making the services flexible to ensure that individual differences are addressed. Finally, further research is needed to understand how service providers view the utility of using classification-based approaches for treatment planning. Even though such strategies appear promising for reducing heterogeneity in a larger clinical population, it is important to ensure that strategies used for treatment planning are appropriately tailored to the clinical environment and how providers make treatment decisions.

Acknowledgments This work was supported by the Department of Veterans Affairs, Veterans Health Administration, Health Services Research and Development Service (IIR 02-283) and the National Institute of Mental Health (MH 74509; MH 79994). The views expressed in this article are those of the authors and do not necessarily represent the views of the Department of Veterans Affairs.

\section{References}

1. Barsevick AM, Whitmer K, Nail LM, Beck SL, Dudley WN (2006) Symptom cluster research: conceptual, design, measurement, and analysis issues. J Pain Symptom Manage 31:85-95

2. Bauer MS, McBride L (2003) Structured group psychotherapy for bipolar disorders. The Life Goals Program. Springer, NY

3. Bauer MS, Crits-Christoph P, Ball WA, Dewees E, McAllister T, Alahi P, Cacciola J, Whybrow PC (1991) Independent assessment of manic and depressive symptoms by self-rating. Scale characteristics and implications for the study of mania. Arch Gen Psychiatry 48:807-812

4. Bauer MS, Vojta C, Kinosian B, Altshuler L, Glick H (2000) The Internal State Scale: replication of its discriminating abilities in a multisite, public sector sample. Bipolar Disord 2:340-346

5. Bauer MS, Kirk GF, Gavin C, Williford WO (2001) Determinants of functional outcome and healthcare costs in bipolar disorder: a high-intensity follow-up study. J Affect Disord 65:231-241

6. Bauer M, Unutzer J, Pincus HA, Lawson WB (2002) Bipolar disorder. Ment Health Serv Res 4:225-229

7. Bauer M, Glenn T, Grof P, Rasgon NL, Marsh W, Sagduyu K, Alda M, Lewitzka U, Sasse J, Kozuch-Krolik E, Whybrow PC (2008) Frequency of subsyndromal symptoms and employment status in patients with bipolar disorder. Soc Psychiatry Psychiatr Epidemiol, in press

8. Blow FC, McCarthy JF, Valenstein M, Austin K, Gillon L (2000) Care for Veterans with Psychosis in the VHA, FY04: 6th Annual Nation Psychosis Registry Report. VA National Serious Mental Illness Treatment Research and Evaluation Center (SMITREC), Ann Arbor

9. Cameron IM, Crawford JR, Lawton K, Reid IC (2008) Psychometric comparison of PHQ-9 and HADS for measuring depression severity in primary care. Br J Gen Pract 58:32-36

10. Cooke RG, Kruger S, Shugar G (1996) Comparative evaluation of two self-report Mania Rating Scales. Biol Psychiatry 40:279283

11. Cooke RG, Robb JC, Young LT, Joffe RT (1996) Well-being and functioning in patients with bipolar disorder assessed using the MOS 20-ITEM short form (SF-20). J Affect Disord 39:93-97

12. Coward-Bucher CE (2008) Toward a needs-based typology of homeless youth. J Adolesc Health 42:549-554

13. Dickerson FB, Boronow JJ, Stallings CR, Origoni AE, Cole S, Yolken RH (2004) Association between cognitive functioning and employment status of persons with bipolar disorder. Psychiatr Serv 55:54-58

14. Dion GL, Tohen M, Anthony WA, Waternaux CS (1988) Symptoms and functioning of patients with bipolar disorder six months after hospitalization. Hosp Commun Psychiatry 39:652-657

15. Fenn HH, Bauer MS, Altshuler L, Evans DR, Williford WO, Kilbourne AM, Beresford TP, Kirk G, Stedman M, Fiore L (2005) Medical comorbidity and health-related quality of life in bipolar disorder across the adult age span. J Affect Disord 86:4760 
16. Gitlin MJ, Swendsen J, Heller TL, Hammen C (1995) Relapse and impairment in bipolar disorder. Am J Psychiatry 152:16351640

17. Gordon AJ, Maisto SA, McNeil M, Kraemer KL, Conigliaro RL, Kelley ME, Conigliaro J (2001) Three questions can detect hazardous drinkers. J Fam Pract 50:313-320

18. Huang JA, Weng RH, Lai CS, Hu JS (2008) Exploring medical utilization patterns of emergency department users. J Formos Med Assoc 107:119-128

19. Judd LL, Akiskal HS (2003) The prevalence and disability of bipolar spectrum disorders in the US population: re-analysis of the ECA database taking into account subthreshold cases. J Affect Disord 73:123-131

20. Keck PE, McElroy SL, Strakowski SM, West SA, Sax KW, Hawkins JM, Bourne ML, Haggard P (2003) 12-Month outcome of patients with bipolar disorder following hospitalization for a manic or mixed episode. Focus 1:44-52

21. Keller MB, Lavori PW, Coryell W, Andreasen NC, Endicott J, Clayton PJ, Klerman GL, Hirschfeld RM (1986) Differential outcome of pure manic, mixed/cycling, and pure depressive episodes in patients with bipolar illness. JAMA 255:3138-3142

22. Kilbourne AM, Bauer MS, Pincus H, Williford WO, Kirk GF, Beresford T (2005) Clinical, psychosocial, and treatment differences in minority patients with bipolar disorder. Bipolar Disord 7:89-97

23. Kilbourne AM, Brar JS, Drayer RA, Xu X, Post EP (2007) Cardiovascular disease and metabolic risk factors in male patients with schizophrenia, schizoaffective disorder, and bipolar disorder. Psychosomatics 48:412-417

24. Kilbourne AM, Lasky E, Pincus HA, Good CB, Cooley S, Basavaraju A, Greenwald D, Fine MJ, Bauer MS (2008) The continuous improvement for veterans in care: Mood Disorders (civic-md) Study, a VA-academic partnership. Psychiatr Serv 59:483-485

25. Klinkman MS (1997) Competing demands in psychosocial care. A model for the identification and treatment of depressive disorders in primary care. Gen Hosp Psychiatry 19:98-111

26. Klonsky ED, Olino TM (2008) Identifying clinically distinct subgroups of self-injurers among young adults: a latent class analysis. J Consult Clin Psychol 76:22-27

27. Kroenke K, Spitzer RL, Williams JB (2001) The PHQ-9: validity of a brief depression severity measure. J Gen Intern Med 16:606613
28. Leon AC, Shear MK, Portera L, Klerman GL (1992) Assessing impairment in patients with panic disorder: the Sheehan Disability Scale. Soc Psychiatry Psychiatr Epidemiol 27:78-82

29. Leon AC, Solomon DA, Mueller TI, Turvey CL, Endicott J, Keller MB (1999) The range of impaired functioning tool (LIFERIFT): a brief measure of functional impairment. Psychol Med 29:869-878

30. Lopez AD, Murray CC (1998) The global burden of disease, 1990-2020. Nat Med 4:1241-1243

31. Macias C, Jones DR, Hargreaves WA, Wang Q, Rodican CF, Barreira PJ, Gold PB (2008) When programs benefit some people more than others: tests of differential service effectiveness. Adm Policy Ment Health 1-12

32. McKibbin C, Patterson TL, Jeste DV (2004) Assessing disability in older patients with schizophrenia: results from the WHODASII. J Nerv Ment Dis 192:405-413

33. Perron BE, Ilgen MA, Hasche L, Howard MO (2008) Service needs of clients in outpatient substance-use disorder treatment: a latent class analysis. J Stud Alcohol Drugs 69:449-453

34. Sevigny EL, Coontz PD (2008) Patterns of substance involvement and criminal behavior: a gender-based cluster analysis of Pennsylvania arrestees. Int $\mathbf{J}$ Offender Ther Comp Criminol 52:435-453

35. Shevlin M, Murphy J, Dorahy MJ, Adamson G (2007) The distribution of positive psychosis-like symptoms in the population: a latent class analysis of the National Comorbidity Survey. Schizophr Res 89:101-109

36. Simon GE, Ludman EJ, Bauer MS, Unutzer J, Operskalski B (2006) Long-term effectiveness and cost of a systematic care program for bipolar disorder. Arch Gen Psychiatry 63:500-508

37. Spilsbury JC, Kahana S, Drotar D, Creeden R, Flannery DJ, Friedman S (2008) Profiles of behavioral problems in children who witness domestic violence. Violence Vict 23:3-17

38. Spitzer RL, Williams JB, Kroenke K, Linzer M, deGruy FV 3rd, Hahn SR, Brody D, Johnson JG (1994) Utility of a new procedure for diagnosing mental disorders in primary care. The PRIME-MD 1000 study. JAMA 272:1749-1756

39. Watzke S, Galvao A, Brieger P (2008) Vocational rehabilitation for subjects with severe mental illnesses in Germany: a controlled study. Soc Psychiatry Psychiatr Epidemiol, in press 